BRE 22177

\title{
Differential effects of chronic partial myelotomies on monoamine levels in cat spinal cord
}

\author{
Kenneth L. Casey ${ }^{1,2,3}$, Thomas J. Morrow ${ }^{2,3}$, L. Cass Terry ${ }^{1,2,3}$ and Ronald Craig ${ }^{3}$ \\ Departments of ${ }^{1}$ Neurology and ${ }^{2}$ Physiology, University of Michigan, Ann Arbor, MI 48109 (U.S.A.) and \\ ${ }^{3}$ Neurology Research Laboratories, Veterans Administration Medical Center, Ann Arbor, MI 48105 (U.S.A.)
}

(Accepted 16 December 1986)

Key words: Cat; Spinal cord; Lesion; Monoamine; Neurotransmitter

\begin{abstract}
The concentrations of 5-hydroxytryptamine (5-HT), norepinephrine (NE) and dopamine (DA) were measured in samples of lumbar and cervical spinal cords from 6 cats with chronic (over 2 months) lesions of the thoracic spinal cord and from 7 unoperated cats. Lesions confined to the dorsal thoracic spinal cord significantly lowered lumbar concentrations of NE, but not 5-HT, compared with control lumbar or matched paired cervical samples. Both NE and 5-HT were significantly reduced by dorsal or ventral lesions that involved tissue ventral to the central canal. Only the largest lesion could be shown to reduce lumbar DA concentration.
\end{abstract}

The supraspinal origins and spinal projections of descending serotonin (5-HT)-, norepinephrine (NE)- and dopamine (DA)-containing pathways have been intensively studied (for review, see Björklund and Skagerberg ${ }^{2}$ ). Although it is known that these pathways have both dorsal and ventral components, and there is general agreement about their origins and terminations, there is no quantitative estimate of the relative contribution of the dorsal and ventral components of each pathway to the innervation of spinal segments. This becomes an important factor in considering the mechanisms by which partial spinal lesions produce changes in spinal cord function. At any specified segmental level, for example, a lesion involving one sector of the spinal cord could selectively interrupt one descending neurotransmitter system while partially or completely sparing other neurotransmitter pathways.

Previous studies have determined quantitatively the regional distribution of one or more of the above neurotransmitters at various spinal segmental levels, but this information does not reveal the relative contribution of specific pathways ${ }^{7,9,11-13,15,17}$. Neuroana- tomical tracing techniques ${ }^{3.8}$ do not provide the necessary quantitaive information. As part of a study on the sensory-behavioral effects of partial thoracic spinal cord transections in the $\mathrm{cat}^{4}$, we wished to determine the nature and degree to which such lesions might differentially affect those descending monoamine systems that may mediate modulation of somatosensory transmission at the spinal cord level ${ }^{1,16}$. Because of the long survival time necessary for behavioral studies, we were able to determine some of the neurochemical effects of chronic partial spinal cord lesions. Some of the results of this study have been briefly presented elsewhere ${ }^{5}$.

Thirteen adult female cats were used in this study. All aspects of animal care and housing and all experimental procedures were conducted in accord with standards and guidelines established by the American Association for the Accreditation of Laboratory Animal Care. The 6 cats used for partial lesion studies each had a complete vertebral laminectomy over spinal cord segments $\mathrm{T}_{6-8}$ while maintained under deep pentobarbital anesthesia $(50 \mathrm{mg} / \mathrm{kg})$ with supplemental doses sufficient to eliminate flexor re-

Correspondence: K.L. Casey, Chief, Neurology Service (127), Veterans Administration Medical Center, 2215 Fuller Road, Ann Arbor, MI 48105, U.S.A. 
flexes. Lesions of the dorsal ( 3 cats) or ventral ( 3 cats) spinal cord were made with iridectomy scissors, pieces of razor blade and the beveled edge of 25 gauge hypodermic needles. The exposed spinal cord was flooded with sterile iced saline immediately before and after creating the lesion. Each cat was given intramuscular antibiotics daily for one week postoperatively. Except for the development of hind limb atrophy, cats with chronic spinal lesions maintained their body weight and normal food consumption, appeared active and alert and responded affectionately to petting and handling. All cats exercised freely outside their cages daily.

Euthanasia for each of the 6 operated cats took place from 2 to 3 months after the placement of the partial myelotomy. At the time of euthanasia, the 6 operated and 7 intact control cats were deeply anesthetized with pentobarbital. Before transcardiac perfusion with a formalin-saline solution, a $2 \mathrm{~cm}$ section that included the lesion was removed for histological analysis and $1 \mathrm{~cm}$ sections of the cervical and lumbar spinal cord were removed and divided into dorsal and ventral halves for biochemical analysis.

After fixation in $10 \%$ formalin-saline, $50 \mu \mathrm{m}$-frozen sections were cut coronally approximately $1 \mathrm{~cm}$ rostral and caudal to the lesion and sagittally through the lesion site. Alternate sections were stained with Cresyl violet and by the Weil-Weigert method for myelin. The location and extent of each lesion was determined from drawings of the projected images of each coronal and sagittal section, noting the areas of gliosis and myelin loss adjacent to each lesion.

For biochemical analysis, weighed tissue samples were sonicated in $0.1 \mathrm{M} \mathrm{HClO}_{4}$ and centrifuged at $12,000 \mathrm{~g}$. After repeating this procedure, the pooled supernatants were analyzed for DA, NE, and 5-HT by HPLC and electrochemical detection as previously described ${ }^{14}$. Briefly, separation was performed on a $5 \mu \mathrm{m} \mathrm{C-18} \mathrm{Biophase} \mathrm{ODS} \mathrm{column.} \mathrm{An} \mathrm{LC-4A} \mathrm{elec-}$ trochemical detector was used with a glassy carbon electrode maintained at $+0.65 \mathrm{~V}$ against an $\mathrm{Ag}-$ $\mathrm{AgCl}$ reference. Monochloroacetic acid buffers $(\mathrm{pH}$ 3.0) were used for the mobile phases of quantitation. Detection limits, defined as a signal/noise ratio greater than 3 with an injection volume of $100 \mu$, were 3 $\mathrm{ng} / \mathrm{g}$ tissue for DA, NE and 5-HT.

Lumbar and cervical concentrations of 5-HT, NE and DA are shown in Fig. 1 for both intact control

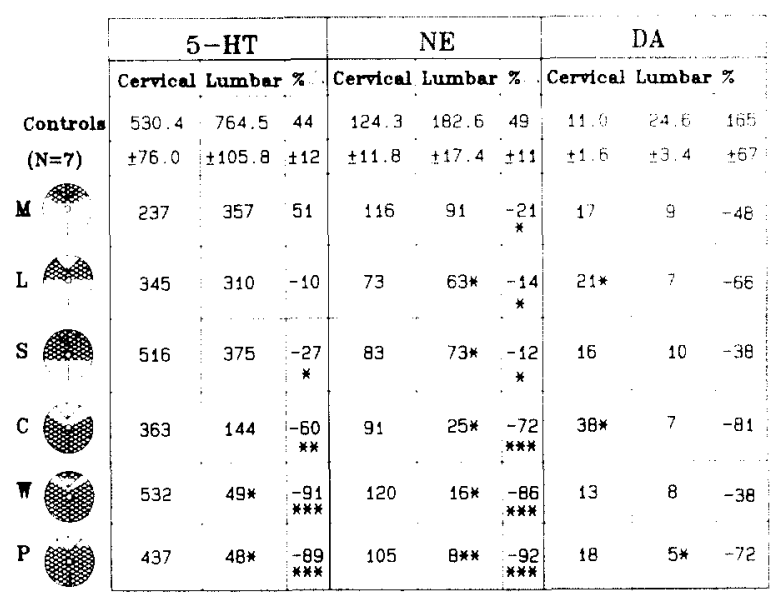

Fig. 1. Neurotransmitter concentrations (ng/g tissue) in samples of whole cat spinal cord. Control values in unoperated cats expressed as mean \pm S.E.M. Percent difference $(\%)$ from cervical concentration computed as lumbar-cervical level/cervical level $\times 100$. Diagrammatic reconstruction of spinal cord lesion of each cat (designated by letter) shown at left margin. Sample values deviating from the control average (top of each column) by 2 S.D. are indicated by 1 asterisk; by 3 S.D., 2 asterisks; by 4 S.D., 3 asterisks.

and lesioned cat spinal cords. Separate control measurements of dorsal and ventral cord sections were taken but are not shown here because a significant dorsal-ventral difference was detected only for NE at the cervical level (dorsal sample: $85.6 \pm 12.0$ (S.E.M.) ng/g; ventral sample: $163 \pm 12.3$ (S.E.M.) $\mathrm{ng} / \mathrm{g} ; t=4.5 ; P<0.01)$. Dorsal and ventral samples were accordingly averaged together in further analy. ses.

In the intact cord, the concentration of each of the monoamines was higher in the lumbar, as compared to cervical, segments. However, this lumbar-cervical difference was siginificant statistically only for $\mathrm{NE}(t=2.41 ; P<0.05)$ and DA $(t=3.62 ; P<0.01)$. The control values of lumbar 5 -HT concentration we obtained are in close agreement with similar measurements from whole spinal cord segments in the $\mathrm{cat}^{6}$ and $\mathrm{rat}^{13,15}$ and are within the range reported by Oliveras et al. ${ }^{12}$. Our measurements of both cervical and lumbar 5-HT and NE are also in accord with previous results obtained from rat, rabbit and cat when differences among species and between whole cord and separate gray and white matter measurements

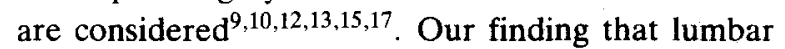
spinal cord levels of both 5-HT and NE in whole spinal cord sections are normally slightly higher than at 
cervical levels is in agreement with the data obtained by others for the rat ${ }^{10,15}$. These lumbar-cervical differences in monoamine concentration may be attributed to differences in the relative amounts of gray and white matter in the cervical and lumbar segments, as suggested by Zivin et al. ${ }^{17}$. The DA concentrations we obtained from the intact spinal cord at the lumbar level are within the range of those reported by Commissiong and Neff ${ }^{7}$ and FleetwoodWalker and Coote ${ }^{9}$ although somewhat lower than those reported by Zivin et al. ${ }^{17}$. Most of these comparative measurements, however, were taken from spinal gray matter and thus are not comparable to measurements from whole spinal cord samples.

Although all thoracic spinal cord lesions were associated with a drop in DA concentration in lumbar segments, only the largest, nearly complete transection resulted in a DA concentration that was significantly below lumbar control levels. Matched pairs of lumbar and cervical samples from the lesioned cats showed that lumbar DA concentrations were lower in each case, but there was no apparent relationship between the size or the location of the thoracic lesion and either the absolute or relative drop in lumbar DA concentration.

In contrast, thoracic spinal cord lesions reduced the lumbar concentrations of both 5-HT and NE in a pattern reflecting the size and location of the lesions. In particular, dorsal lesions that did not extend ventral to the central canal (cats $\mathrm{M}$ and $\mathrm{L}$ ) significantly lowered lumbar NE concentrations but left lumbar 5HT concentrations unchanged. The comparatively low cervical concentration of 5-HT in cat $\mathrm{M}$, although well within 2 S.D. of the control mean, may have precluded demonstrating a relative drop in lumbar 5-HT concentration in the matched samples. However, an even larger lesion restricted to the dorsal spinal cord (cat $\mathrm{L}$ ) failed to produce a significant relative or absolute drop in lumbar 5-HT concentration while significantly lowering both relative and absolute lumbar NE levels. A larger dorsal lesion that extended just ventral to the central canal (cat S) also

1 Basbaum, A.I. and Fields, H.L., Endogenous pain control mechanisms: review and hypothesis, Ann. Neurol,, 4 (1978) 451-462.

2 Björklund, A. and Skagerberg, G., Descending monoaminergic projections to the spinal cord. In B. Sjölund and A. reduced lumbar NE concentrations but additionally resulted in a small but significant drop in lumbar 5HT concentrations relative to the matched cervical sample. This result can be compared with the effect of a similarly sized ventral lesion (cat $\mathrm{C}$ ) that spared the dorsal pathways that were sectioned in cats $\mathrm{L}$ and $\mathrm{M}$ and produced a significant relative drop in lumbar 5-HT and a significant absolute and relative reduction of lumbar NE levels (cat C). Although it is not possible to draw conclusions based on a comparison of single samples, the lesion in cat $C$ involved more of the intermediate area in the plane of the central canal where the concentration of 5-HT- and NE-containing endings is especially high ${ }^{2,17}$. Very extensive ventral lesions that spared only the dorsolateral funiculi produced marked and significant reductions in lumbar concentrations of both NE and 5-HT (cats W and P).

The failure of all but the largest thoracic spinal cord lesions to reduce significantly the lumbar concentration of DA is in agreement with the evidence ${ }^{7}$ for an intraspinal dopaminergic neural population that would not be affected by the transection of descending DA projections. However, the relatively low levels of spinal DA and the variability of the cervical-lumbar differences among the paired samples may have prevented detecting the effect of interrupting the known diencephalospinal DA system ${ }^{2}$.

Our results show that chronic dorsal thoracic spinal cord lesions are more likely to affect NE, as compared to 5-HT concentrations, below the level of the lesion. Although both NE and 5-HT descending systems are known to have dorsal and ventral components, our findings suggest that, at least at the thoracic level, the dorsal 5-HT pathway contributes less to lumbar 5-HT levels than the dorsal NE pathway contributes to lumbar NE concentrations. Ventral 5-HT and NE spinal pathways apparently both contribute substantially to spinal concentrations of each of these putative neurotransmitters. These results are important in interpreting the behavioral, physiological and neurochemical effects of spinal cord lesions.

Björklund (Eds.), Brain Stem Control of Spinal Mechanisms, Elsevier, Amsterdam, 1982, pp. 55-88.

3 Carlsson, A., Falck, B., Fuxe, K. and Hillarp, N.-A., Cellular localization of monoamines in the spinal cord, Acta Physiol. Scand., 60 (1963) 112-119. 
4 Casey, K.L., Hall, B.R. and Morrow, T.J., Effect of spinal cord lesions on responses of cats to thermal pulses. Pain. Suppl. 1 (1981) 130.

5 Casey, K.L., Morrow, T.J. and Terry, L.C., Pain suppression persists after interruption of descending serotonergic and noradrenergic pathways in cat spinal cord, Abstract no. 85, Fourth General Meeting, American Pain Soc., Chicago, IL, 1983, Abstracts and Meeting Information, 1983, p. 84.

6 Clineschmidt, B.V., Pierce, J.E. and Lovenberg, W., Tryptophan hydroxylase and serotonin in spinal cord and brain stem before and after spinal transection. $J$. Neuro. chem., 18 (1971) 1593-1596.

7 Commissiong, J.W. and Neff, N.H., Current status of dopamine in the mammalian spinal cord. Biochem. Pharmacol., $28(1979)$ 1569-1573.

8 Dahlström. A. and Fuxe. K., Evidence for the existence of monoamine-containing neurons in the central nervous system. I. Demonstration of monoamines in the cell bodies of brain stem neurons. Acta Physiol. Scand., 62. Suppl. 232 (1964) $1-55$.

9 Fleetwood-Walker, S. and Coote, J.H., Contribution of noradrenaline-, dopamine-, and adrenaline-containing axons to the innervation of different regions of the spinal cord of the cat,. Brain Research, 206 (1981) 95-106.

10 Magnusson, T. . Effect of chronic transection on dopamine, noradrenaline and 5-hydroxytryptamine in the rat spinal cord, Naunyn-Schmiedeberg's Arch. Pharmacol., 278 (1973) 13-22
11 Naftchi. N.E., Demeny, M., DeCriscito, V., Tomasulat. J.J., Flamm, E.S. and Campbell, J. B., Biogenic amine con centrations in traumatized spinal cords of cats. J. Neure surg., 40 (1974) 52-57.

12 Oliveras, J.L., Bourgoin, S., Hery, F., Besson, J.M. and Hamon, M., The topographical distribution of serotonergic terminals in the spinal cord of the cat: biochemical mapping by the combined use of microdissection and microassaly procedures, Brain Research, 138 (1977) 393-406.

13 Pang, I.-H. and Vasko, M.R., Effect of depletion of spinal cord norepinephrine on morphine-induced antinociception, Brain Research, 371 (1986) 171-176.

14 Terry, L.C. and Craig, R., Cysteamine effects on monoamines. dopamine- $\beta$-Hydroxylase, and the hypothalamicpituitary axis, Neuroendocrinology, 41 (1985) 467-47.5.

15 Vasko, M.R., Pang. I.-H. and Vogt, M., Involvement of 5 hydroxytryptamine-containing neurons in antinociception produced by injection of morphine into nucleus raphe magnus or onto spinal cord. Brain Research. 306 (1984) $341-348$.

16 Yaksh, T.L. and Hammond, D.L., Putative transmitters of descending systems-studies of local administration and liberation. In B. Sjölund and A. Björklund (Eds.), Brain Stem Control of Spinal Mechanisms, Elsevier, Amsterdam, 1982. pp. $473-492$.

17 Zivin, J.A., Reid, J.L., Saavedra, J.M. and Kopin, T.J., Quantitative localization of biogenic amines in the spinal cord, Brain Research, 99 (1975) 293-301 